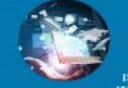

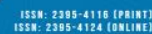

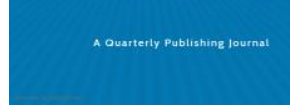

Prajwal Khupat ${ }^{1}$ prajwalkhupat5@gmail.com

Usha Barapatre ushabarapatre460@gmail.com

Nikita Khadatkar nikhitakhadatkar@gmail.com

Sneha Badge ${ }^{1}$ snehabadge2@gmail.com

Pooja Meharkar ${ }^{1}$ poojameharkar8@gmail.com

Shweta Bhelonde ${ }^{2}$ shwetabhelonde@gmail.com

${ }^{1}$ Student, Department of Computer Engineering, Smt. Radhikatai Pandav college of Engineering, Nagpur, India

${ }^{2}$ Assistant Professor, Department of Computer Engineering, Smt. Radhikatai Pandav college of Engineering, Nagpur, India

\title{
IOT Based ECG Monitoring and Analysis System with Machine Learning
}

Abstract - Internet of Things is that the new revolution that's about to impact each side of our lives. web of Things (IOT) is the new technology which incorporates the gathering of Sensors, Actuators, methods and alternative development boards (e.g., Raspberry pi, Arduino etc.) to gather the info from different sources relying upon domain of application, process the inhabitable data before causing to distant cloud or mobile application depending upon the requirement. The foremost promising application of IOT is within the field of health care sector. In some countries, folks still don't have access to quality health facilities due to different barriers. The physical distance between patient and clinic is one among the foremost reasons. This paper studies the applying of IOT in health care domain and a system is planned to observe the ECG of the distant patient. Smart and reasonable health care is more and more in demand to satisfy the requirements of the growing human population and medical expenses. Graph monitoring is an extensively studied and applied approach to identification heart disease.

Keywords - IOT, ECG sensor, machine learning, web application

\section{INTRODUCTION}

By 2020, because of the extraordinary development within the net of Things (IOT), it'll be conceivable to debate fifty billion connected Devices over the Internet. Sensors worn on the body are among the assorted devices that observe individual diseases. Recently there has been a growing interest in wearable sensors and variety of latest parts are financially accessible to detect action. For clinical

Research Article

First Online on - 10 July 2021

(C) 2021 RAME Publishers

This is an open access article under the CC BY 4.0 International License https://creativecommons.org/licenses/by/4.0/

Cite this article - Prajwal Khupat, Usha Barapatre, Nikita Khadatkar, Sneha Badge, Pooja Meharkar, Shweta Bhelonde, Vaishali C. Asole Samiksha S. Thul, "IOT Based ECG Monitoring and Analysis System with Machine Learning", International Journal of Computational and Electronic Aspects in Engineering, RAME Publishers, vol. 2, issue 3, pp. 51-56, 2021

https://doi.org/10.26706/ijceae.2.3.20210602 use, the observation of long patients and managers was additionally considered. The 2 drivers of this innovation are the range of IOT-based info and cloud-based research. Initiated the birth of portable graphical record observation frames. For example, the good telephone-based methodology for perceptive biological signals is bestowed by a elaborate review of varied numerous approaches in healthcare was carried out. A cloud-based transportable ECG observation aid has been introduced. This will differentiate between ECG signals with the assistance of a non-intrusive device and transmit the signal to the organizer exploitation remote streaming methods comparable to Bluetooth or Zigbee. However, nearly all current pictures cannot operate while not a complicated cellular phone used as a receiver and processor for ECG information. an out of this world result on daily PDA use. 
Also, to enhance all the fundamentals of the good terminal in operation system, it takes an incredible effort to bit by bit improve the transportable application. portable devices for cypress trees. This proved info is sent to the knowledge set related to successfulness restoration. Measurable referral calculations will place this current patient information with a large information base from numerous patients and supply the specialist with a range of suggestions. The portable graphical record monitor can transfer data on to the IOT cloud via wireless local area network while not the necessity for a mobile terminal. Here, the cloud-based ECG watching system design of the net of Things (IOT) is proposed. an occasional power transportable graphical record watching system exploitation PSoC is employed to accumulate the ECG signal from the human body. High-speed, superior WICED that transfers knowledge on to the Amazon net Services (AWS) IOT cloud. Compared to Bluetooth or Zigbee, WiFi offers higher data speeds and bigger coverage areas. Most of the process is shipped on the server side. OT allows RFID, BLE, Wi-Fi and alternative device networks so computers will understand the globe for themselves. OT Cloud permits users to own convenient and timely access to ECG data. communications protocol and MQTT servers are provided in the IOT cloud. This system unceasingly monitors the physical signs of patients comparable to blood pressure, ECG, $\mathrm{SpO} 2$ in addition as relevant environmental indicators and offers four totally different knowledge transfer modes that balance the necessity for treatment and also the necessities for communication and computing resources enforced a sample image to produce an summary of the system to give. This watching system fulfills the essential general health care needs for heart condition and also takes into consideration the price to make sure that the final mode is as economical as doable. In addition, it may also be combined with time period analysis algorithms to judge patients. Health standing and warning of possible attacks that can create widespread treatment smarter.

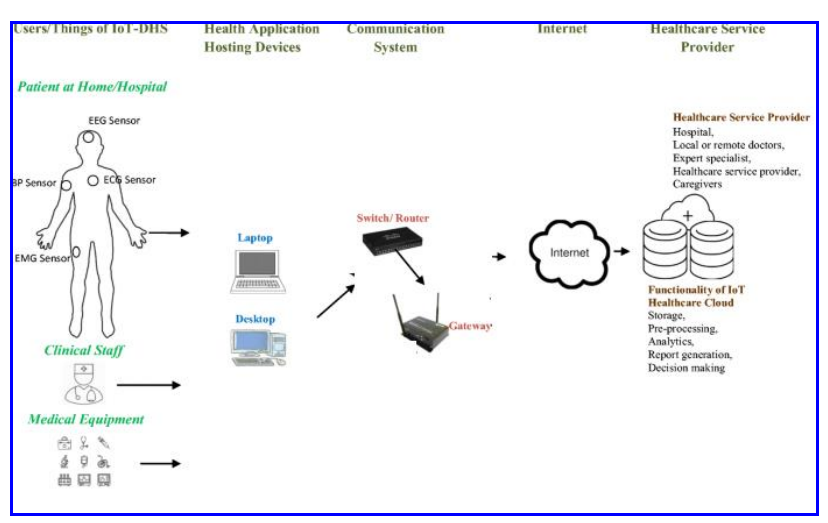

Figure 1. General Working of System

Basically, above diagram represent the overall structure of the purpose system in this system it consists of several parts shown in figure in the system it consists one IOT Device in the IOT device consist the hardware such as NODEMCU and ECG sensor. NODEMCU responsible to make connection with web application and provide an internet connectivity to device and there is one web application which manage all the patient and doctor information and their ecg report the web app can be accessible through laptop desktop and mobile and this web app is hosted at server.

As figure 1 shows the flow of system basically whenever sister/doctor will connect the IOT device to patient body then it will calculate the ecg for certain time once it done it will upload the ECG data to the server thought the internet and server will apply the machine learning novel algorithm and analyze the ECG data and determine whether patient ECG is normal or abnormal an in case of abnormal the system will automatically book an appointment with doctor through AI.

\section{BRIEF STUDY}

Shola et. al. was proposed for the gathering of the readings of various important indications of the patients. Later send the readings to the doctor or the individual about the health condition. In this project MQTT communication is used to send the data to the cloud platform. The patient's vital sense is transmitted with the help of the pictorial representation [1].

Mehmet used a wearable sensor which keeps the track of the patient's heart beat and the blood level. The patient 
who has the critical health situations can be continuously monitored using these techniques. If there is a fluctuation of the health in the patient then the information/details of his health conditions are sent to the family members or the doctor through the mail or twitter notifications. The purpose is to give medical treatment as soon as possible in case of the heart diseases. So, there will be an increased chance of the survival of the patients [2].

Syed et. al. proposed the system for the victims of mass disasters and emergencies. MEDTOC is a real time component used for the holistic solution. The proposed system sends the details of the effected victims to the doctor or to the central database about their health condition even before the arrival of the patient. By this the medical staff can prepare the necessary treatment and can operate as soon as the patient is arrived. In this the data of the patient which is transferred is stored without the identification of the patient. But this project can only be useful if the disaster area has the cellular network. If the cellular network is having issues, then the alternate connectivity such as Wi-Fi can be directed in the future [3].

\section{METHODOLOGY}

Architecture is a key factor that sets our proposed system apart from other previously proposed systems that use body sensors to monitor heart rate and provide ECG data. In our system, an ECG sensor is connected to the body, as shown in Figure 1 Patients can regularly monitor their health so that they can contact their doctor. Even doctors can pull the reports from the Mongo DB database. Add the patient ID that will be saved when the patient comes for an exam. This happens when the patient ID is entered, the EMR trigger and the measured values are updated via the web connection. Pulse sensor and EKG are connected to the Raspberry pi via ADS1115 analog readings. Sensors such as pulse and ECG are converted into digital samples before they are used by the Arduino Collector App. The advantages of this model for reading ECG and pulse sensors are: 1 Since a patient's disease diagnosis is mainly based on a series of tests and their overall results, including the vital signs recorded at the time of the consultation with OPD, do not manually record the vital signs which leads to a wrong diagnosis. Therefore, our IOT-based cloud platform offers the measurement of important functions such as pulse and ECG values, which are automatically recorded in the patient's system EMR at the time of patient visits. This method enables healthcare providers to minimize errors due to the manual entry of important data. Our IOT-based cloud platform enables healthcare providers to be seamlessly mobile and to conduct mass exams where many patients are examined, for example: NGO camps.

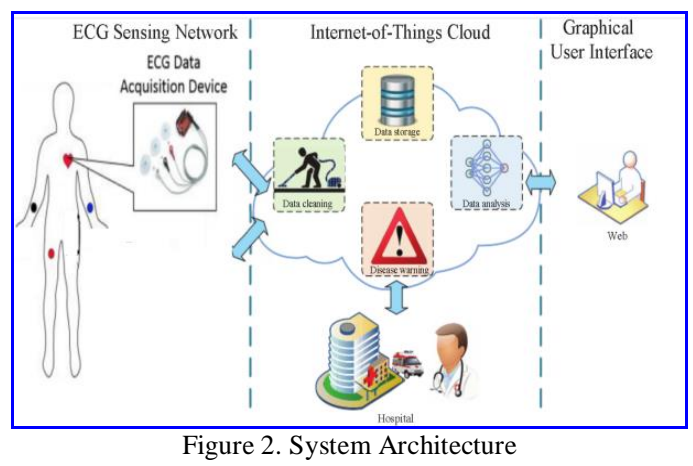

A. Algorithm

Step 1. Start

Step 2. Gather 30 seconds ECG data from device.

Step 3. Analyze ECG data.

Step 4. Calculate BPM ( blood per minute).

$\mathrm{BPM}=($ Single wave time* $30 \mathrm{sec} / \mathrm{min}) /$ No.of waves.

Step 5. If BPM > 80 then "abnormal"

If $\mathrm{BPM}<80$ then "abnormal"

Else "normal".

Step 6. Calculate inter beat interval

(time interval between two beat).

Step 7. Select the two or more R-waves, then find the value of standard deviation of RR interval. SDRR $=\sqrt{ }($ RR interval $1-R R$ interval 2$)+\sqrt{ }(R R$ interval 2 - RR interval 3) +..... $\sqrt{(}(\mathrm{RR}$ interval 3 - RR interval $n)$.

Step 8. Calculate breathing rate between 30 seconds.

Breathing rate $=$ No. of breathing per second $* 60 / 30$

Step 9. Stop.

A novel set of rules is verified on this system. The Python coronary heart fee evaluation toolkit is known as HeartPy and is beneficial for reading coronary heart fee information gathered through HeartPy in noisy 
environments. It is a module for coronary heart fee evaluation in Python. It commenced out as a natural Python implementation for reading physiological information captured in naturalistic driving and biking experiments. The module alternatives up a discrete coronary heart fee sign and generates time area and frequency area measurements which are regularly discovered with inside the medical literature. The Pan-Tompkins Algorithm is the maximum extensively used QRS complicated detector for monitoring many coronary heart conditions, inclusive of detecting arrhythmias. This technique may want to offer top detection overall performance with excessive pleasant scientific ECG waveform information. However, the numerous kinds of noise and artifacts found in an ECG sign produce negative pleasant ECG sign information. For this reason, the overall performance of Pan-Tompkins-primarily based totally QRS detection techniques the usage of negative pleasant ECG alerts ought to be in addition investigated. the QRS complicated of general ECG information inclusive of noise-loaded ECG alerts. The acoustic strain check information and the MIT-BIH arrhythmia information. The overall performance of the set of rules changed into then analyzed and presented. This article demonstrates the cap potential of Pan-Tompkins algorithms to system noisy ECG alerts. The set of rules is output as a heartbeat wave diagram. Algorithm will examine the enter ECG information and constitute the output in under format.

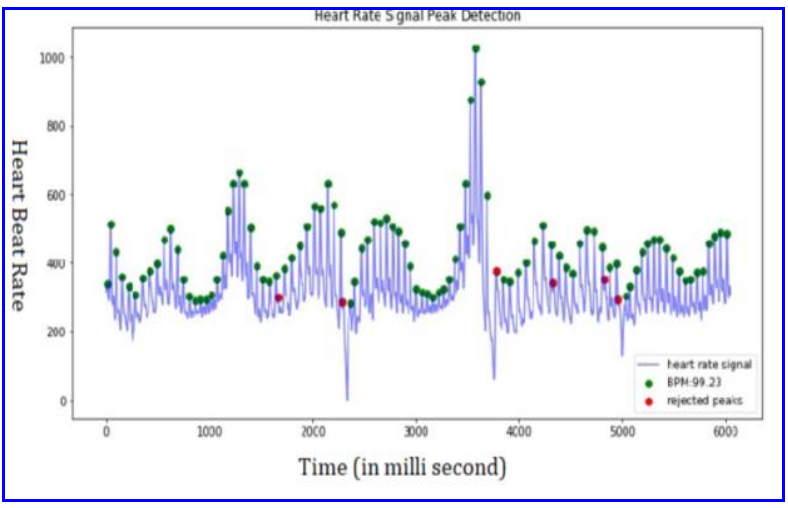

Figure 3. ECG Graph

a) Heart beats per minute, BPM

b) Interbeat interval, IBI

c) Standard deviation if intervals between adjacent beats, SDNN d) Standard deviation of successive differences between adjacent R-R intervals, SDSD

e) Root mean square of successive differences between adjacent R-R intervals, RMSSD

f) Proportion of differences between R-R intervals greater than 20ms, 50ms, PNN20, PNN50

g) Median absolute deviation, MAD

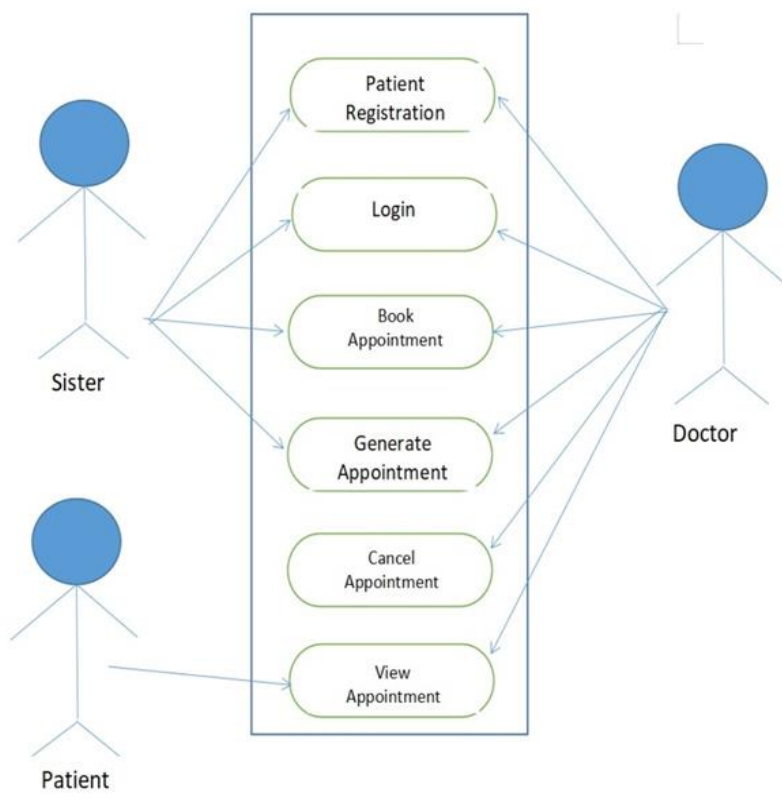

Figure 4. UML Diagram

\section{B. Technique Used}

Software Requirement

1. Front End : HTML, CSS

2. Back End : Python, Flask, Arduino

3. Domain : IOT, Cloud Computing, Machine Learning,

Hardware Requirement

1. Processor : i3 or grater

2. RAM $: 4 \mathrm{~GB}$ or greater

3. Hard Disk : $50 \mathrm{~GB}$ or greater

4. Connectivity: LAN or WIFI

IOT Components Requirement

\section{ESP8266}

2. Wi-Fi Module

3. AD8232 ECG Sensor Kit

\section{RESUlT AND DisCUSSION}

The result of this work included 3 patients. The ECG monitoring started age from $22 \mathrm{yr}$. old. The chart 
mentioned ECG report is normal. In this system depends on the heart rate and heart rate range under $80 \mathrm{bpm}$ is normal range. That's why this patients report is normal and $80 \mathrm{bpm}$ above range that patients report is abnormal.

TABLE I

ECG REPORT OF THREE PATIENTS

\begin{tabular}{|c|c|c|c|c|c|}
\hline Name of Patient & $\begin{array}{l}\text { Bloodper } \\
\text { minute }\end{array}$ & Inter Beat Intervd & $\begin{array}{l}\text { Standard Deviotion } \\
\text { of NN } \\
\text { interval }\end{array}$ & $\begin{array}{l}\text { Stundard Deviotion } \\
\text { of RR } \\
\text { interval }\end{array}$ & $\begin{array}{l}\text { Breothi } \\
\text { ngrote }\end{array}$ \\
\hline $\begin{array}{l}\text { Bhoskar } \\
\text { Mhodatkor }\end{array}$ & 70 & 1. 29719281805882 & 58 & 72 & B \\
\hline $\begin{array}{l}\text { Niḱto } \\
\text { Nhodatkor }\end{array}$ & 79 & $\begin{array}{c}130828699063425 \\
9\end{array}$ & 57 & 92 & R \\
\hline $\begin{array}{l}\text { Revanto } \\
\text { Nhodatkor }\end{array}$ & 69 & $\begin{array}{c}0.133351771940859 \\
7\end{array}$ & 56 & 86 & $\theta$ \\
\hline
\end{tabular}

In this purposed there are 6 different modules described below.

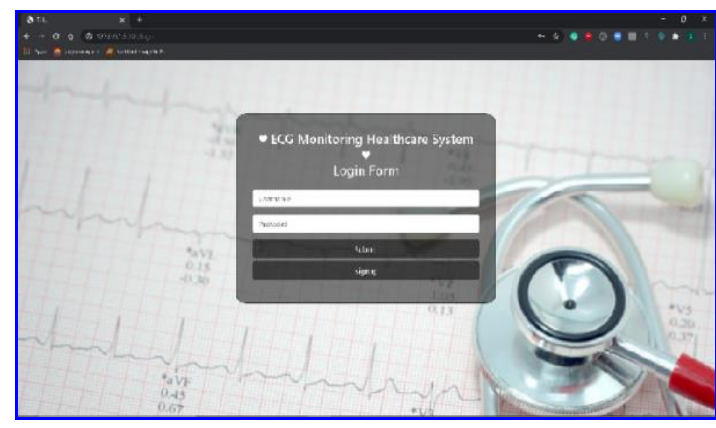

Figure 4. Login Module

1. Login Module: In this module doctor or sister are able to login into web application.

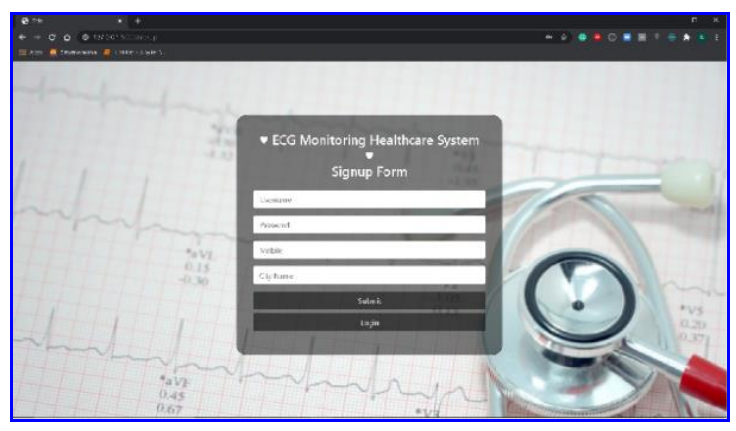

Figure 6. Registration Module

2. Registration Module: From this module doctor or nurses are able to register new patient details in the system.

3. ECG Monitoring Module: In this module, ECG sensor will attach to patient and wait for 30 seconds for monitoring the ECG report and upload ECG report to our cloud.

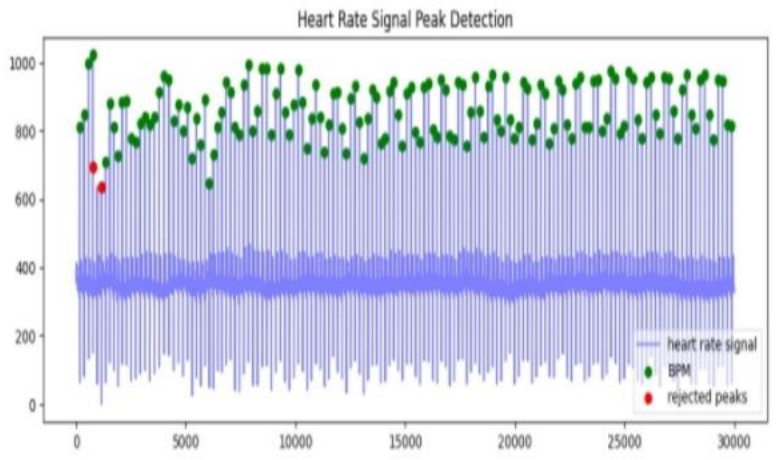

Figure 7. ECG Graph

4. ECG Analysis Module: After successfully uploading the data of ECG report from IOT to server then. In this module our machine learning model will analyze the ECG report and make prediction whether patient is suffering from any disease or not and show some heart related factor details.

5. Machine Learning Algorithm Training: In this module we will train our machine learning model to predict whether patient is suffering from the heart disease or not.

\begin{tabular}{|c|c|c|c|c|c|c|c|}
\hline \multicolumn{8}{|c|}{ Patient Heart ECG Report } \\
\hline $\begin{array}{l}\text { Blood Per } \\
\text { minute }\end{array}$ & \multicolumn{2}{|c|}{ Inter Beat Interval } & \multicolumn{2}{|c|}{$\begin{array}{l}\text { Standard Deviation of NN } \\
\text { Interval }\end{array}$} & \multicolumn{2}{|c|}{$\begin{array}{l}\text { Standard Deviation of RR } \\
\text { Interval }\end{array}$} & $\begin{array}{l}\text { Breathing } \\
\text { Rate }\end{array}$ \\
\hline 70 & \multicolumn{2}{|c|}{2.1934811661247484} & \multicolumn{2}{|l|}{70} & \multicolumn{2}{|r|}{42} & 14 \\
\hline \multicolumn{8}{|c|}{ Result: Normal ECG Found } \\
\hline \multicolumn{8}{|c|}{ Doctor Appointment : Not Required } \\
\hline Patient Name & Patient Surname & & Patient Email & Patien & obile & Doctor Appointed & Appointment Date \\
\hline Nikita & Khadatkar & nikhit: & thadatkar@gmail.com & 8999 & & & \\
\hline \multicolumn{8}{|c|}{ Download Report File } \\
\hline
\end{tabular}

Figure 8. ECG Report

6. Book Doctor Appointment: In this module, it will book anointment of patient with doctor automatically of patient suffering from heart disease.

\section{FUTURE SCOPE}

In future this technology we can more improvement in the machine learning algorithm part we can train algorithm with more data and also can create a IOT device with multiple widget such as display oximetry sensor and can add multiple sensors and make it multi-function. 


\section{CONCLUSIONS}

Our system is inexpensive, mobile, and valid with real subjects, albeit they're largely healthy subjects. Such a system can save lots of greenbacks as a result of medical hospitals can have vast bills for heart failure, eliminating the requirement for frequent hospital visits. By saving cash for the patient and saving hospital resources, the doctors time may be dedicated to more important events, further as victimization hospital emergency resources. therefore far, our system is in a position to gather sensing element knowledge, send data from iot to the server net application, and draw period data from all sensors within the smartphone application.

\section{REFERENCES}

[1] Mehmet Tastan, IOT based Wearable Smart Health Monitoring System, Celal Bayar University Journal of Science, 2018, 343-350.

[2] Syed Misbahuddin, JunaidAhmed Zubairi,AbdulRahman Alahdul, Muhammad Arshad Malik, IOT- based Ambulatory Vital Signs Data Transfer System, Hindawi Journal of Computer Networks and Communications, 2018, 8 pages.
[3] Duarte Dias, Joao Paulo Silva Cunha, Wearable Health Devices-Vital Sign Monitoring Systems and Technologies, MDPI Journal, 2018, 18(8):2414.

[4] K.Satwik, N.V.K.Ramesh, SK.Reshma, Estimation and Monitoring of Vital Signs in the Human Body by using Smart Device, International Journal of Innovative Technology and Exploring Engineering, 2019, 1035-1038.

[5] Faisal Jamil, Shabir Ahmad, Naeem Iqbal, Do-HyeunKim, towards a Remote Monitoring of Patient Vital Signs Based on IOT-Based Block chain Integrity Management Platforms in Smart Hospitals, MDPI Journal, 2020, 20, 2195.

[6] Mehak Chhabra, Manik Kalsi, "Real time ECG monitoring system based on Internet of Things (IOT), IJSRP journal, 2017, 547-550.

[7] Zhe Yang, Qihao Zhou, Lei Lei, Kan Zheng, “An IOT-cloud Based Wearable ECG Monitoring System for Smart Healthcare", Journal of Medical System 40(12):286, 2016.

[8] Jorge Gomez, Byron Oviedo, Emilio Zhuma, "Patient Monitoring System Based on Internet of Things", Computer Science 83(2016), 90-97.

[9] Jeevan Karle, Haftu T Reda, Soo Y Shin, “An architecture for smart health monitoring system based on Fog Computing", Journal of Communication, 2017, 12(4), 228233. 